\title{
Saccadic facilitation by modulation of microsaccades in natural backgrounds
}

\author{
Petra Sinn • Ralf Engbert
}

Published online: 19 February 2011

(C) The Author(s) 2011. This article is published with open access at Springerlink.com

\begin{abstract}
Saccades move objects of interest into the center of the visual field for high-acuity visual analysis. White, Stritzke, and Gegenfurtner (Current Biology, 18, 124-128, 2008) have shown that saccadic latencies in the context of a structured background are much shorter than those with an unstructured background at equal levels of visibility. This effect has been explained by possible preactivation of the saccadic circuitry whenever a structured background acts as a mask for potential saccade targets. Here, we show that background textures modulate rates of microsaccades during visual fixation. First, after a display change, structured backgrounds induce a stronger decrease of microsaccade rates than do uniform backgrounds. Second, we demonstrate that the occurrence of a microsaccade in a critical time window can delay a subsequent saccadic response. Taken together, our findings suggest that microsaccades contribute to the saccadic facilitation effect, due to a modulation of microsaccade rates by properties of the background.
\end{abstract}

Keywords Eye movements $\cdot$ Microsaccade $\cdot$ Saccade latency $\cdot$ Background texture $\cdot$ Saccadic facilitation effect

\section{Introduction}

The visual exploration of stationary scenes is based on active sampling, using eye movements, because of the limited visual angle that is mapped to the fovea - that is, the

Electronic supplementary material The online version of this article (doi:10.3758/s13414-011-0107-9) contains supplementary material, which is available to authorized users.

P. Sinn $\cdot$ R. Engbert $(\square)$

Department of Psychology, University of Potsdam,

Haus 14, Raum 4.02, Karl-Liebknecht-Str. 24-25,

14476 Potsdam OT Golm, Germany

e-mail: Ralf.Engbert@uni-potsdam.de region of highest acuity within the human retina. Here, we focus on the interaction of saccades, rapid eye movements for the generation of shifts between different image patches, and microsaccades, the fastest component of fixational eye movements (Engbert 2006; Martinez-Conde, Macknik, \& Hubel, 2004), which are generated involuntarily to counteract retinal fading (Engbert \& Mergenthaler, 2006; MartinezConde, Macknik, Troncoso, \& Dyar, 2006). Microsaccades represent small-amplitude ballistic movements (during fixation) that follow the saccadic main sequence (Zuber, Stark, \& Cook, 1965). Most of our knowledge of saccadic latency is obtained from laboratory trials involving brief fixations on simple stimuli embedded in uniform backgrounds. However, White, Stritzke, and Gegenfurtner (2008) demonstrated that saccadic latency, one of the most important measures of visual processing, is critically influenced by background texture. In this study, we investigated the potential relations among saccadic latency, microsaccadic activity, and background properties.

Typical natural scenes sampled from our environment are complex but share inherent statistical regularities. Mandelbrot (1975) pioneered the computational study of statistical self-similarity by the estimation of fractal properties. For example, the Fourier spectrum of typical natural scenes can be characterized by a power law of the form $1 / f^{\beta}$, where the exponent $\beta$ is found to be in the range of $1.0 \pm 0.2$ (Field, 1987; Simoncelli \& Olshausen, 2001; van der Schaaf \& van Hateren, 1996).

How are stimuli identified within complex natural scenes that provide extensive masking? One approach to this problem is based on the observation of saccadic latencies toward emerging stimuli. Interestingly, such latencies can be reduced considerably when stimuli are masked by a natural background (White et al., 2008). With a series of control experiments, it was ruled out that the effect is not a simple consequence to the signal-to-noise ratio. Therefore, White et 
al. concluded that the saccadic facilitation effect might be caused by specific preactivation of the saccadic circuitry for critical stimuli masked by their natural environment.

During visual fixation, while we are preparing for a rapid response to an imminent sensory signal, our eyes perform microsaccades with a typical rate of 1 to 2 per second (for an overview, see Engbert, 2006; Martinez-Conde et al., 2004). In general, microsaccade rate undergoes a characteristic temporal signature of decreased rate (microsaccadic inhibition) after a display change (Engbert, 2006; Engbert \& Kliegl, 2003). Using a replication of the original work by White et al. (2008), we investigated whether background texture influences microsaccade rate. Modulations of microsaccade statistics are potentially important for the saccadic facilitation effect, since saccadic latencies can be delayed by microsaccades in a critical time window shortly before saccade onset (Rolfs, Laubrock, \& Kliegl, 2006). Therefore, we were interested in investigating whether uniform backgrounds can induce less microsaccadic inhibition than can natural backgrounds and, consequently, prolong the latencies of upcoming saccades, due to more microsaccades in the critical time window.

\section{Method}

Participants Thirty-one participants were paid $€ 7$ or received study credits for their attendance. All the participants were naive as to the nature of the study. They had normal or corrected-to-normal visual acuity and ranged from 18 to 31 years of age ( 22.5 years on average). Our experiment was performed in accordance with the ethical standards laid down in the 1964 Declaration of Helsinki, and the participants gave their informed consent prior to their inclusion in the study.

Task, apparatus, and design Stimuli were displayed on a 22-in. FT/LCD monitor at a refresh rate of $60 \mathrm{~Hz}$ and a resolution of 1,680 $\times 1,050$ pixels. At a viewing distance of $70 \mathrm{~cm}$, eye movements were measured using an EyeLink II system (SR Research, Osgoode/Ontario, Canada) with a sampling rate of $500 \mathrm{~Hz}$ and a spatial resolution better than $0.01^{\circ}$. At the beginning of the experiment and after each block of 10 trials, a standard 9-point calibration (followed by a validation) was performed. Measurements were done without a chinrest; however, participants were asked to prevent head movements during the trials.

The design of the experiment was taken from White et al. (2008). The target was a stationary, vertically oriented Gabor patch $\left(S D=0.7^{\circ}, 1 \mathrm{cpd}\right)$, which appeared randomly $8^{\circ}$ left or right of the central fixation spot (black circle, $d=$ $0.2^{\circ}$ ). The background was a uniform gray background, a $1 / f$ noisy structured background (100 images produced using
MATLAB Software, $50 \%$ in contrast), or a natural scene (taken from a total of 100 images, 1,024 × 1,024 pixels, black and white). Average luminance values were $33.5 \mathrm{~cd} / \mathrm{m}^{2}$ for uniform background, $30.4 \mathrm{~cd} / \mathrm{m}^{2}$ for $1 / f$ background, and $40.5 \mathrm{~cd} / \mathrm{m}^{2}$ for natural images as background condition. Background conditions were presented in randomized order. The experiment was programmed using Python and the VisionEgg package (Straw, 2008).

In a first task, individual contrast thresholds for $75 \%$ correct responses to uniform gray and $1 / f$ backgrounds were determined for each participant in a detection task using the adaptive QUEST algorithm (Billock, 2000; King-Smith, Grigsby, Vingrys, Benes, \& Supowit, 1994; Watson \& Pelli, 1983). Starting contrast values were $\log T=-2.8$ for uniform and $\log T=-1.8$ for $1 / f$ backgrounds. Every trial started with a keypress. A randomly chosen background and the fixation point were presented for a random period of between 800 and $1,200 \mathrm{~ms}$, followed by a period in which the target appeared to the left or to the right of the fixation spot. Participants were instructed to respond as quickly as possible, using left or right computer keys corresponding to the target position, but to suppress saccades to the targets. Overall, 30 trials (15 trials per background) were performed in three blocks of 10 trials.

The second part of the experiment was a saccadic task consisting of 11 blocks. To avoid extra movements caused by pressing a key, 10 trials ( 5 trials in the last block) were presented continuously, one after another, with a $500-\mathrm{ms}$ break. The randomly chosen background (uniform gray, $1 / f$ structured, or natural) and the fixation spot were present for a random period of between 800 and 1,200 ms, followed by a gap period (i.e., the fixation point disappeared) for $200 \mathrm{~ms}$, followed by a period in which the target (using the contrast estimated in the first experiment) appeared to the left or right of the fixation point. Participants were instructed to respond with a saccadic eye movement to the target as promptly and accurately as possible. Overall, 35 trials for each background condition were performed (i.e., 105 trials per participant).

Data analysis We analyzed eye movements from trials with correct saccades and without any blinks. Saccades and microsaccades were detected using the same algorithm (Engbert \& Kliegl, 2003; Engbert \& Mergenthaler, 2006). Saccades were defined as epochs lasting for $6 \mathrm{~ms}$ or more, in which an elliptic threshold of the two-dimensional velocity vector was surpassed (threshold multiplier: 30). Latencies were estimated as time differences between target onset and the onset of the first saccade. The saccadic facilitation effect was characterized by the average difference between the latencies for uniform and structured backgrounds. Microsaccades were detected using a threshold multiplier of 5. The continuous evolution of the microsaccade rate was computed by applying a causal window (e.g., Dayan \& Abbott, 2001), 
which produced an optimized estimate of the saccadic rate variation as a function of time.

\section{Results}

We observed shorter saccadic latencies for structured backgrounds than for uniform backgrounds. The average latencies were $390 \mathrm{~ms}(S E= \pm 19 \mathrm{~ms})$ for uniform background, $254 \mathrm{~ms}$ $(S E= \pm 9 \mathrm{~ms})$ for $1 / f$ backgrounds, and $225 \mathrm{~ms}(S E= \pm 6 \mathrm{~ms})$ for natural backgrounds (Fig. 1). The size of the average facilitation effect was about $150 \mathrm{~ms}, F(2,60)=77.6, p<$ $\left.10^{-4}\right)$. A test of within-subjects contrasts was significant for $1 / f$ versus uniform background, $F(1,30)=69.0, p<10^{-4}$, as well as for natural versus uniform background, $F(1,30)=$ 89.2, $p<10^{-4}$ ). Therefore, our implementation of the paradigm by White et al. (2008) replicated the saccadic facilitation effect; however, the facilitation effect turned out to be stronger than in the original study.

Next, we investigated microsaccade rates in time frames of $200 \mathrm{~ms}$ and distinguished three characteristic epochs. During the first epoch (baseline, interval 1, from -400 to $-200 \mathrm{~ms}$ relative to target onset), microsaccade rates were indistinguishable across background conditions (Fig. 2). We observed an effect of the display change at gap onset, which resulted in a reduction of the microsaccade rate after the disappearance of the fixation stimulus in the second epoch (microsaccadic inhibition, interval 2, from -200 to $0 \mathrm{~ms}$ relative to target onset), which replicates the microsaccadic inhibition effect from an earlier study on the impact of display changes on microsaccade rates (Engbert \& Kliegl, 2003).

The third epoch (response preparation, interval 3, from 0 to $+200 \mathrm{~ms}$ ) was related to the saccadic response. Here, we observed a second microsaccadic inhibition, followed by an increase of the microsaccade rate, which might be interpreted as premotor activity due to the imminent saccadic response.

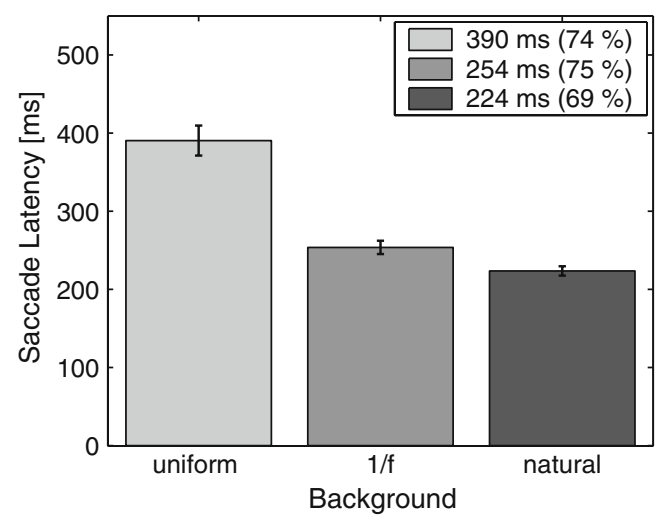

Fig. 1 Average saccadic latency for different backgrounds. Error bars represent the standard errors of the means. Percentage values in the legend represent the proportions of correct saccadic responses

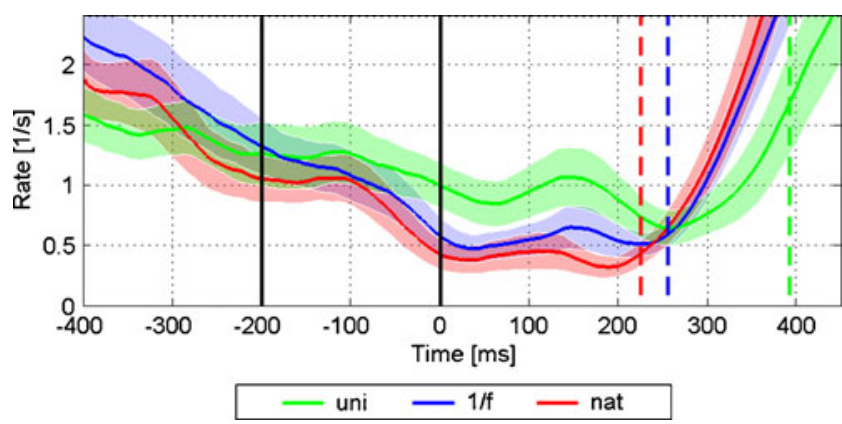

Fig. 2 Temporal evolution of microsaccade rates locked to target onset. The beginnings and ends of the gap periods are indicated by vertical black lines (bold); average saccadic response times are added to the plot by the vertical dashed lines (color). Standard errors (obtained from interindividual differences) for the rate curves are given by the shaded areas. Rate modulations were strongly influenced by background condition

In summary, there was no general effect of background condition on microsaccade rate (interval 1); however, we found differences in the strength and duration of the microsaccadic inhibition effect between background conditions. The reduction of the microsaccade rate was stronger (and observed for a longer time window) for natural and $1 / f$ backgrounds than for uniform backgrounds in the second and third intervals.

To investigate whether the microsaccadic rate modulations potentially contribute to the saccadic facilitation effect, we separated trials into categories with microsaccades (latency $L_{\mathrm{MS}}$ ) and without microsaccades (latency $L_{0}$ ). We determined the last microsaccade before the saccadic response in each trial, because microsaccades shortly before the saccadic response are likely to produce the largest inhibitory effect on latencies (Rolfs et al., 2006). Figure 3 illustrates the dependence of the ratio of $L_{\mathrm{MS}} / L_{0}$ for intervals 1 to 3 defined

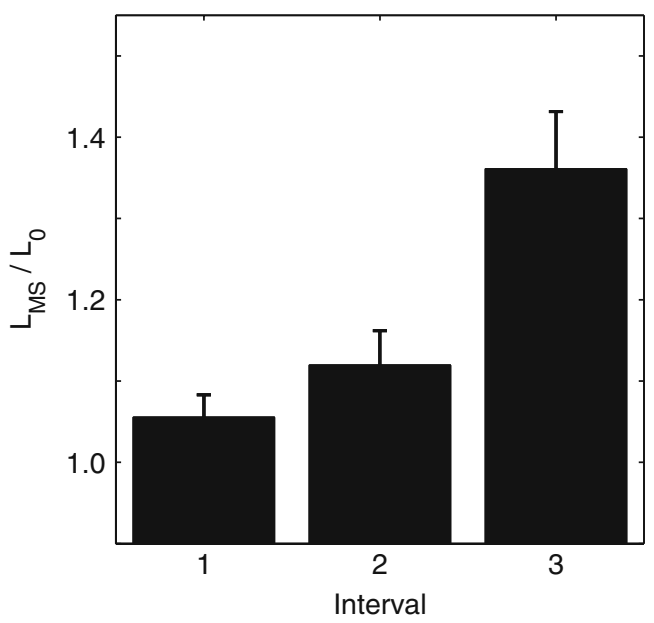

Fig. 3 Inhibitory effects of microsaccades on saccadic responses. An increased ratio of the latency with $\left(L_{\mathrm{MS}}\right)$ and without $\left(L_{0}\right)$ a microsaccade during three different subsequent intervals was observed. Error bars represent the standard errors of the means 
above. We observed the inhibitory effect of microsaccades when we compared intervals 1 and 2 (this analysis was not considered on the level of individual participants, due to lack of statistics). We found that the occurrence of microsaccades during the fixation period (interval 1) had less influence on the saccadic latencies than did microsaccades occurring in the inhibition period (interval 2). Latencies increased up to $30 \%$ whenever a microsaccade occurred. Our results lend support to the hypothesis that microsaccades inhibit the saccadic response if they occur shortly before the saccadic response. Thus, the absence of microsaccades contributes to the saccadic facilitation effect.

\section{Discussion}

We were interested in the possible contribution of microsaccades to the saccadic facilitation effect (White et al., 2008) in natural backgrounds. We replicated the facilitation effect and recorded eye movements in order to investigate fixationrelated saccadic activity (microsaccades). First, we did not observe a main effect of background texture on microsaccade rate. Thus, the control of fixational eye movements is not specifically dependent on background texture. Second, we observed a microsaccadic rate modulation, which was related to a display change, as has been observed in earlier studies and across a number of experiments (for an overview, see Engbert, 2006). Third, the size and duration of the rate modulation effect were clearly influenced by background texture. The inhibition of microsaccades in a uniform background is delayed by $40 \mathrm{~ms}$, and the rate, at the minimum, of the inhibition period is twice as high as the rate for structured backgrounds. Fourth, microsaccades interfere with saccadic reactions and delay latencies. As a consequence, saccadic responses are inhibited by microsaccades occurring shortly before the saccades (see Rolfs et al., 2006).

Our results indicate that variations of microsaccade rates, which are induced by different background conditions, might contribute to the saccadic facilitation effect in natural backgrounds (White et al., 2008). To exclude the alternative hypothesis that the microsaccadic rate modulations are caused by prolonged latencies (and not vice versa), we performed a control experiment with $100 \%$ visibility to achieve equal latencies across all conditions (see the supplemental materials, Experiment 2). As a result, we consistently found a higher microsaccade rate for uniform backgrounds (during interval 2) than for natural backgrounds. Microsaccadic rate effects can be modulated in the absence of the facilitation effect, and, therefore, the direction of causality is likely to go from microsaccadic rate modulation to the facilitation effect, and not vice versa.

The microsaccadic inhibition effect induced by display changes (Engbert \& Kliegl, 2003) turned out to be less pronounced for uniform backgrounds. In a second control experiment, we investigated the role of the gap period (see the supplemental materials, Experiment 3). As a result, both the microsaccadic rate effect and the saccadic facilitation effect were replicated. Hence, the gap period is not critical for the interaction of background structures, microsaccade rates, and saccadic latencies.

In summary, there is a higher prevalence of microsaccades during the microsaccadic inhibition epoch for a uniform background than for natural (or 1/f) backgrounds. As a consequence, the occurrence of a microsaccade shortly before a saccadic response is more likely, which, on average, delays the saccadic response (Rolfs et al., 2006).

Why should there be more microsaccades in uniform background conditions? Microsaccades are generated to enhance activity of the retinal receptor systems and to counteract retinal bleaching (Martinez-Conde et al., 2004, 2006). Moreover, microsaccades are triggered whenever slow components of fixational eye movements do not produce sufficient retinal slip (Engbert \& Mergenthaler, 2006). From these considerations, fixational eye movements are ineffective with a uniform background, due to missing patterns that could stimulate retinal receptor systems as a result of eye motions. To further investigate this hypothesis, we carried out an additional experiment with different structured backgrounds (see the supplemental materials, Experiment 4). Most important, we presented band-pass filtered backgrounds that contained statistical patterns without the characteristic $1 / \mathrm{f}$ decay of spatial correlations. In this condition, we retained the modulations of microsaccade rates, but without a saccadic facilitation effect. Thus, the enhanced activity of microsaccades in a uniform background, as compared with a natural background, might be interpreted as a response of the fixational eye movement system to reduced (or missing) background structure.

Author Note We thank Karl R. Gegenfurtner for comments on the manuscript and Konstantin Mergenthaler for valuable discussions. This work was supported by Deutsche Forschungsgemeinschaft (Grant EN 471/3-1, being part of DFG Research Unit 868, "Computational modeling of behavioral, neural, and cognitive dynamics").

Open Access This article is distributed under the terms of the Creative Commons Attribution Noncommercial License which permits any noncommercial use, distribution, and reproduction in any medium, provided the original author(s) and source are credited.

\section{References}

Billock, V. A. (2000). Neural acclimation to $1 / f$ spatial frequency spectra in natural images transduced by the human visual system. Physica D, 137, 379-391. doi:10.1016/S0167-2789(99)00197-9

Dayan, P., \& Abbott, L. F. (2001). Computational neuroscience: Computational and mathematical modeling of neural systems. Cambridge: MIT Press. 
Engbert, R. (2006). Microsaccades: A microcosm for research on oculomotor control, attention, and visual perception. Progress in Brain Research, 154, 177-192. doi:10.1016/S0079-6123(06)54009-9

Engbert, R., \& Kliegl, R. (2003). Microsaccades uncover the orientation of covert attention. Vision Research, 43, 1035-1045. doi:10.1016/S0042-6989(03)00084-1

Engbert, R., \& Mergenthaler, K. (2006). Microsaccades are triggered by low retinal image slip. Proceedings of the National Academy of Sciences, 103, 7192-7197. doi:10.1073/pnas.0509557103

Field, D. J. (1987). Relations between the statistics of natural images and the response properties of cortical cells. Journal of the Optical Society of America, 4, 2379-2394. doi:10.1364/JOSAA.4.002379

King-Smith, P. E., Grigsby, S. S., Vingrys, A. J., Benes, S. C., \& Supowit, A. (1994). Efficient and unbiased modifications of the quest threshold method: Theory, simulations, experimental evaluation and practical implementation. Vision Research, 34, 885-912. doi:10.1016/0042-6989(94)90039-6

Mandelbrot, B. B. (1975). Stochastic models for the earth's relief, the shape and fractal dimension of coastlines, and the number area rule for islands. Proceedings of the National Academy of Sciences, 72, 2825-2828. doi:10.1073/pnas.72.10.3825

Martinez-Conde, S., Macknik, S. L., \& Hubel, D. H. (2004). The role of fixational eye movements in visual perception. Nature Reviews. Neuroscience, 5, 229-240. doi:10.1038/nrn1348
Martinez-Conde, S., Macknik, S. L., Troncoso, X. G., \& Dyar, T. A. (2006). Microsaccades counteract visual fading during fixation. Neuron, 49, 297-305. doi:10.1016/j.neuron.2005.11.033

Rolfs, M., Laubrock, J., \& Kliegl, R. (2006). Shortening and prolongation of saccade latencies following microsaccades. Experimental Brain Research, 169, 369-376. doi:10.1007/s00221-005-0148-1

Simoncelli, E. P., \& Olshausen, B. A. (2001). Natural image statistics and neural representation. Annual Review of Neuroscience, 24, 1193-1216. doi:10.1146/annurev.neuro.24.1.1193

Straw, A. D. (2008). Vision Egg: An open-source library for realtime visual stimulus generation. Frontiers in Neuroinformatics, 2(4), 1-10. doi:10.3389/neuro.11.004.2008

van der Schaaf, A., \& van Hateren, J. H. (1996). Modelling the power spectra of natural images: Statistics and information. Vision Research, 36, 2759-2770. doi:10.1016/0042-6989(96)00002-8

Watson, A. B., \& Pelli, D. G. (1983). QUEST: A Bayesian adaptive psychometric method. Perception \& Psychophysics, 33, 113-120.

White, B. J., Stritzke, M., \& Gegenfurtner, K. R. (2008). Saccadic facilitation in natural backgrounds. Current Biology, 18, 124-128. doi:10.1016/j.cub.2007.12.027

Zuber, B. L., Stark, L., \& Cook, G. (1965). Microsaccades and the velocity-amplitude relationship for saccadic eye movements. Science, 150, 1459-1460. doi:10.1126/science.150.3702.1459 